\title{
Rougir / devenir rouge : l'expression linguistique du langage corporel et ce qu'elle laisse inférer dans un genre textuel
}

\section{Rougir / became red: the linguistic expression of the body language and what it lets to infer in a textual genre}

\author{
Teresa Muryn \\ Université Pédagogique de Cracovie \\ teresa.muryn@up.krakow.pl
}

\begin{abstract}
The subject of this article is part of research trying to identify in a textual genre lexico-syntactic constructions with generic markers value. The analysis focuses on the linguistic expression of the manifestation of the blushing body in two textual genres: the detective novel and the romance novel. The aspectual value of these constructions may be related to the textual genre in which they appear because it seems to guide the inferential mechanism in the discovery of implicit information.
\end{abstract}

Keywords: body language, lexico-syntactic construction, textual genre, verbal aspect, inference

L'analyse ci-présente s'inscrit dans le cadre de recherches du groupe Disem ${ }^{1}$ qui postule l'existence d'une matrice lexico-syntaxique d'un genre textuel. Elle serait composée de constructions lexico-syntaxiques propres à un genre et obligatoires pour la reconnaissance du genre. La recherche s'inspire de travaux similaires dans le cadre de la phraséologie dite étendue (F. Grossman, D. Legallois, I. Novakova, A. Tutin) et de recherches linguistiques sur le genre textuel (F. Rastier, J.-M. Adam, H. Grzmil-Tylutki).

\footnotetext{
${ }^{1}$ T. Muryn, M. Niziołek, A. Hajok, W. Prażuch, K. Gabrysiak.
} 
Le corpus sur lequel se fonde cette étude se compose de romans policiers en français venant d'auteurs reconnus, aussi bien français qu'étrangers : Gaston Leroux, Maurice Leblanc, Conan Doyle, Georges Simenon, Fred Vargas, Agatha Christie, Jean-Christophe Grange, Arnaldur Indridason, Maxime Chattam, Dorothy L. Sayers, Harlam Coben, Jean-Claude Izzo, Pierre Magnan, Boileau-Narcejac, Exbrayat, et d'autres ; du corpus «Harlequin » de romans sentimentaux, des données réunies dans Narodowy Korpus Języka Polskiego et des données d'Internet obtenues à l'aide du moteur de recherche Google.

Prenant en compte le principe qu'il existe des traits distinctifs pour un genre textuel on a pu identifier le roman policier comme un genre autonome faisant partie du discours littéraire. Il devrait donc se caractériser par l'existence d'unités textuelles obligatoires le distinguant d'autres genres. Pour les extraire, il s'est révélé indispensable non seulement de morceler le texte en fragments obligatoires du point de vue de la totalité du genre textuel, mais aussi de morceler les fragments obtenus en unités plus basses. $\mathrm{Si}$, par exemple, l'interrogatoire participe obligatoirement à la reconnaissance du roman policier comme genre, il se compose lui-même de plusieurs éléments qui le constituent et qui s'expriment de façon différente dans le dialogue et dans le récit. Pourtant certaines constructions lexico-syntaxiques semblent avoir un caractère stable et récurrent, elles apparaissent dans la plupart des romans analysés. Etant donné qu'il existe plusieurs variantes formelles véhiculant la même information, il s'est avéré nécessaire de les identifier au niveau conceptuel. C'est ainsi que la recherche du groupe se concentre actuellement sur l'identification d'une unité, appelée motif ${ }^{2}$ conceptuel, qui peut être réalisée par une ou plusieurs variantes lexico-syntaxiques parmi lesquelles seulement celles qui ont un caractère récurrent peuvent être classées comme éléments constitutifs de la matrice lexicosyntaxique du genre. Nous présentons dans cet article les premiers résultats de l'analyse du motif " langage du corps » sur l'exemple « rougir-émotion » dans le roman policier et dans le roman sentimental. La recherche n'est pas terminée, mais elle semble autoriser déjà à quelques conclusions. Il s'est avéré, par exemple, que la question de la valeur aspectuelle des prédicats participant à la construction du motif conceptuel « rougir-émotion » joue un rôle très important dans la reconstruction de ce motif dans le texte.

Nous nous sommes concentrée sur le problème de la communication nonverbale pour deux raisons: d'abord, parce que les travaux de psychologues sur le langage du corps (entre autres ceux de Paul Ekman (2018)) ont révélé, dans les relations sociales, le rôle d'une bonne interprétation de la mimique faciale, des gestes et du comportement. Les résultats de ces recherches ont été adaptés, par exemple, dans les procédures d'interrogatoire aidant l'enquêteur à repérer le mensonge. Et même si certaines thèses, telle la détection du mensonge, n'ont pas été

\footnotetext{
${ }^{2}$ Il existe plusieurs définitions de la notion de motif (entre autres celles de D. Longrée et S. Mellet, D. Legallois, I. Novakova). Nous présentons celle qui a été élaborée par le groupe de recherches Disem.
} 
confirmées dans la pratique, l'idée de savoir lire sur le visage s'est inscrite dans la mémoire commune et fonctionne comme stéréotype. D'autre part, parmi les réactions involontaires du corps, certaines semblent affecter les interactions humaines plus que d'autres : c'est le cas de rougir qui peut même atteindre le stade d'une maladie (érythrophobie). Ici aussi, il existe des stéréotypes de situations favorisant cette réaction et des stéréotypes de personnes qui en sont touchées. «Au cours de l'activité humaine, et plus particulièrement dans l'acte de communication, le corps se transforme en vecteur des attributs de la culture humaine et des normes sociales. La culture s'incarne dans le corps » (Krejdlin, 2008, p. 14).

Parmi ces signes donc, il existe ceux qui peuvent être contrôlés et d'autres qui échappent au contrôle du locuteur. Si les premiers (par exemple, l'expression du visage: sourire, bâiller, se mordre les lèvres, etc.), d'origine naturels, peuvent devenir conventionnels, donc utilisables de façon consciente dans la communication (Wierzbicka, 1999), les seconds sont des réactions inconscientes du corps (rougir, pâlir, trembler, etc.); ils ont un caractère indiciel et doivent être interprétés contextuellement. Ce qui est perçu dans ce dernier cas, c'est une manifestation physiologique qui est due à une émotion, l'origine de cette émotion reste à découvrir. Il est donc intéressant de savoir, en premier lieu, quels sont les moyens linguistiques disponibles pour décrire le phénomène et, en deuxième lieu, de savoir si (et comment) les auteurs de romans policiers et de romans sentimentaux l'exploitent au premier plan pour marquer la succession des faits et à l'arrière-plan pour la construction des personnages (Weinrich, 1973).

\section{LA NOTION DE MOTIF CONCEPTUEL}

Nous partons du principe qu'il existe dans les langues naturelles deux niveaux : 1) un niveau conceptuel, sémantique, composé d'un ensemble des concepts et d'un ensemble des règles et 2) un niveau formel, d'expression, composé d'un ensemble d'expressions assignées aux concepts et de règles de leur combinatoire. Le niveau sémantique est universel, tandis que le niveau d'expression est idiomatique. La structure sémantique est complète, la structure formelle qui la représente ne l'est pas obligatoirement : il n'existe pas de symétrie entre les deux niveaux. Cela veut dire qu'à une structure sémantiquement complexe peut équivaloir une structure formelle simple et à l'inverse, une structure formelle complexe peut représenter une structure sémantique simple.

Dans une analyse contrastive, surtout quand les phénomènes linguistiques que l'on compare proviennent de langues divergeant au niveau de leurs systèmes, le caractère universel du niveau sémantique le prédispose à jouer un rôle de tertium comparationis : un niveau auquel on peut se référer pour établir une équivalence entre des phénomènes comparés. 
Le recours à la notion de motif conceptuel semble obligatoire quand une unité autonome dépasse les limites d'une structure sémantique polyprédicative dont les arguments sont sémantiquement impliqués. Il s'agit d'une unité de rang supérieur définissable sur le niveau textuel, sous-jacente à un segment de texte, composée de plusieurs éléments dont chacun peut devenir autonome, mais qui, sans être impliqués, forment un tout. La réalisation du motif se reconnaît au niveau textuel : c'est une unité textuelle composée de segments qui lui assurent la cohérence. Ainsi, le motif conceptuel « rougir-émotion » se compose de trois segments obligatoires.

Le premier segment réfère à la manifestation de l'émotion chez un expérienceur dont la peau du visage a changé en rouge. On peut le paraphraser comme suit :

La peau du visage d'un $x$ est devenue rouge.

Le deuxième segment véhicule l'information sur la source de ce changement d'état :

La peau du visage d'un $x$ est devenue rouge sous l'effet d'une émotion.

Or, comme les émotions, sont, elles-mêmes, des réactions provoquées par la confrontation à une situation et à l'interprétation de la réalité, le troisième segment du motif doit donc référer à la source (cause) de l'émotion :

La peau du visage d'un $x$ est devenue rouge sous l'effet d'une émotion provoquée par un état de choses.

Ces trois segments sont obligatoires (et en même temps suffisants) pour que le motif conceptuel « rougir-émotion » appartienne au domaine du langage corporel. Quant à sa construction, il comprend deux structures causales dans le rapport de subordination. Dans la version chronologique, il s'agit d'un rapport de cause à effet : un état de choses cause une émotion chez un $X$, ce qui entraîne le fait que la peau de son visage devient rouge, par exemple :

L'idée de déballer tout à l'heure des cadeaux de Noël lui a fait tellement plaisir qu'il en a rougi. / Myśl, że zaraz będzie rozpakowywat świąteczne prezenty, sprawiła mu taka przyjemność, że aż poczerwieniat.

Dans la version explicative, l'ordre de l'apparition d'éléments obligatoires de la structure est inversé, de l'effet à la cause : la peau du visage d'un $\mathrm{X}$ devient rouge sous l'effet d'une émotion causée par un état de choses, par exemple :

Il a rougi de plaisir à l'idée de déballer tout à l'heure des cadeaux de Noël. I Poczerwieniat z przyjemności na myśl, że zaraz będzie rozpakowywat świąteczne prezenty. 


\section{LA VALEUR ASPECTUELLE DES PRÉDICATS D'ÉMOTION - MANIFESTATION. LE CAS DE ROUGIR/DEVENIR ROUGE}

L'explicitation du premier segment du motif « rougir-émotion » est assurée par des expressions qui présupposent le changement d'état. C'est surtout le cas du verbe rougir, de la construction devenir rouge et de leurs variantes aspectuelles (ou de synonymes tels que s'empourprer ou rosir qui n'apportent qu'une valeur intensive aux précédents et se comportent comme ces derniers).

En analysant la valeur aspectuelle de ces expressions, nous nous référons à la théorie de S. Karolak présentée dans ses nombreux travaux publiés entre 1991 et 2005. Selon l'auteur, l'aspect est un phénomène universel dont l'étude doit se situer au niveau sémantique. Il s'agit donc d'une catégorie sémantique, un trait inhérent du concept. Cette définition permet d'étendre l'analyse aspectuelle sur des catégories morphologiques traditionnellement exclues d'une telle analyse comme des noms et des adjectifs mais aussi de chercher des indicateurs de la valeur aspectuelle dans un contexte plus large, au moins dans une phrase. S. Karolak (2001a) postule aussi que la catégorisation de verbes selon le critère aspectuel doit se baser sur le critère sémantique, le verbe n'étant qu'une représentation formellement simple du concept qui peut se révéler une unité aspectuellement complexe. Le verbe peut donc véhiculer ce que S. Karolak appelle une configuration aspectuelle : une combinaison d'aspects simples, continu (imperfectif) et non continu (perfectif), avec une dominante (continue ou non continue) responsable de l'organisation de son entourage phrastique.

Selon cette théorie le verbe rougir appartient à la catégorie des verbes inchoatifs. Cette configuration contient deux éléments : l'un, perfectif (non continu) qui domine la structure et qui marque le début de l'état et le deuxième, dominé, imperfectif (continu), qui indique un nouvel état. D'après S. Karolak (2001, p. 638) il n'existe pas une différence aspectuelle entre un verbe inchoatif et un verbe causatif (qu'il appelle résultatif). Ce qui les distingue, c'est la nécessité d'expliciter la composante causative pour des verbes résultatifs, tandis que la position de cette composante est effacée dans le cas de verbes inchoatifs. C'est justement pour cette raison que la structure thème-rhème dans les deux cas est différente : contrairement aux résultatifs, dans les inchoatifs c'est le patient (ici : expérienceur) qui devient le thème de la communication (Ciszewska, 2002, p. 48). Dans les résultatifs, la cause est explicite, dans les inchoatifs elle est inférée. Pour ces derniers, elle peut être identifiée sur la base d'un savoir extralinguistique et/ou de données contextuelles. La structure causale peut enfin être restituée dans les constructions fondées sur les verbes inchoatifs quand ils se combinent avec l'opérateur causatif faire (faire rougir).

La valeur inchoative peut se manifester de différentes façons, entre autres, par un radical verbal imperfectif associé au morphème grammatical, p.ex. du passé simple, exposant de la perfectivité (il rougit) ou par différentes constructions 
combinant les verbes à valeur momentanée avec des noms ou adjectifs (il se sentit rougir, il commença à rougir, il se mit à rougir, il devint rouge, il se fit rouge, etc.).

A partir de la configuration en question, il est possible d'en dériver d'autres, à dominante imperfective, et plus complexes encore. Elles envisagent la manifestation de l'émotion comme un processus en cours. Cette configuration, dite télique, s'exprime à travers un morphème grammatical à valeur imperfective (p.ex. imparfait - il rougissait), à l'aide du gérondif (en rougissant), ou bien à l'aide de constructions à valeur imperfective (le rouge qui monte aux yeux) etc.

Pour toutes ces expressions, quand on prend comme critère un point de référence, elles se divisent en deux groupes : celles qui focalisent le moment de la cause qui coïncide avec le moment du début de l'état (il rougit, il se sentit rougir) et celles qui se concentrent sur l'état résultant ((devenu) rouge, il a rougi, en rougissant, il se sentait rougir, etc.). Cette différence s'avère très importante dans une analyse textuelle puisqu'elle assure la progression textuelle sur la base d'une bonne reconnaissance de données explicites et inférées permettant de restituer le motif conceptuel sous-jacent.

En polonais, qui est une langue aspectuelle, les verbes inchoatifs du type analysé se caractérisent, du point de vue de leur structure formelle, morphémique, d'une complexité qui révèle leur nature sémantique: la base dérivationnelle renvoie à l'aspect imperfectif propre à un état tandis que l'affixe dérivationnel représente la dominante perfective du début de l'état coïncidant avec la cause non spécifiée de cet état. C'est ainsi que s'interprète le verbe poczerwienieć, où le préfixe po-ajouté au verbe imperfectif intransitif czerwienieć sert d'exposant de l'aspect momentané. Le cas du verbe zaczerwienić się est plus complexe : le préfixe $z a$-ajouté au verbe imperfectif transitif czerwienić lui donne la valeur perfective tandis que le pronom pseudo-réfléchi się bloque la possibilité d'expliciter la cause de l'état. Selon W. Śmiech (1986) le préfixe po- dans le cas analysé a une valeur «purement aspectuelle » (1986, p. 27) tandis que $z a$ - hérite du verbe zaczać (commencer) sa valeur inchoative (1986, p. 39). On pourrait donc établir que le premier est équivalent à la version résultative devenu rouge/a rougi et le second à l'inchoatif "pur» rougir. Il existe aussi des constructions inchoatives du type stać się, zrobić się : stat się czerwony jak krew ; zrobit się czerwony jak burak (Kacprzak, 2013, p. 36), mais elles sont relativement rares.

\section{L'EXPLICITATION TEXTUELLE DU MOTIF CONCEPTUEL « ROUGE-ÉMOTION »}

Rappelons le motif conceptuel « rouge-émotion » qui est censé, d'après nous, organiser le texte :

La peau du visage d'un $x$ devient rouge sous l'effet d'une émotion provoquée par un état de choses. 
L'explicitation textuelle du motif :

A. Peut se réduire à son segment principal, celui qui décrit la réaction corporelle en question (la peau du visage d'un $x$ devient rouge), par exemple Pierre rougit.

B. Peut être complétée d'une information véhiculée par le deuxième segment, celui qui porte sur l'émotion-source de la réaction corporelle (la peau du visage d'un $x$ devient rouge sous l'effet d'une émotion), par exemple Pierre rougit de colère.

C. Porte sur les trois segments du motif conceptuel (la peau du visage d'un $x$ devient rouge sous l'effet d'une émotion provoquée par un état de choses), par exemple $A$ ce discours, Pierre rougit de colère.

\subsection{L'explicitation textuelle du segment : réaction corporelle (A)}

Les structures inchoatives dérivées des structures résultatives, causales, bloquent la position ouverte à la cause. Les expressions qui les réalisent ont, bien évidemment, la même propriété : elles fonctionnent comme autonomes sans expliciter la cause du nouvel état. La partie principale du motif peut donc fonctionner dans une phrase autonome :

Il leva les yeux et sourit. Ah bon ? Et si j'insiste? Sylvie rougit. Quelle idiote! (Harlequin)

Monsieur Bordenave est devenu tout rouge et il a commencé à siffler par le nez. (Goscinny)

Sylvie se sentit rougir jusqu'à la pointe des cheveux. (Harlequin)

Je me mis à rougir.

Alison se redressa, rougissante, mais Rod ne se troubla pas. (Harlequin)

Pater spojrzat na czterdziestolatka $z$ wypiekami na twarzy. Mężczyzna poczerwieniat jeszcze bardziej i skiną głowa. (M. Czubaj; M. Krajewski)

Właśnie zaczęła się czerwienić, gdy jej spojrzenie padło na zwisającego mu z ust papierosa. (M. Gable)

\subsection{L'explicitation textuelle de deux segments : réaction corporelle + l'émotion source de la réaction corporelle (B)}

L'explicitation de l'émotion, le deuxième segment du motif conceptuel « rougirémotion » est tout à fait possible dans ces constructions, mais il s'agit d'un élément adjoint, non obligatoire, non impliqué, donc effaçable. E. Pilecka (2010, p. 51-52) constate pourtant que rougir dans son rôle de verbe intensifieur présente "un parallélisme de comportement» avec les verbes supports : "l'association entre le verbe intensifieur et le nom qu'il intensifie fait état de certaines préférences et 
idiosyncrasies ». On peut rougir de confusion ou de honte mais on ne rougit pas de bonheur ou d'amour. Pourtant, selon l'auteure, « la construction est loin d'être figée étant donné que le même verbe peut apparaître avec plus d'un nom : rougir de honte/plaisir/envie/colère/confusion/aise/gêne...». Toutes ces constructions apparaissent fréquemment aussi bien en polonais qu'en français :

Elle dut se rendre compte de son trouble, car elle rougit d'embarras, croisant les bras dans un geste dérisoire pour couvrir sa poitrine. (Harlequin)

Les joues de Kate rougirent sous l'effet de l'indignation et de la révolte. (Harlequin)

Il est devenu rouge comme un coq. On ne savait pas si c'était de honte ou de rage.

Puis elle retira presque aussitôt sa main en rougissant de confusion sous son regard étonné. (Harlequin)

Aż poczerwieniał z bezsilnej złości.

Kobieta zaczerwienita się z zadowolenia.

Pourtant il n'est pas nécessaire que l'expression explicitant le segment de l'émotion suive le verbe ou la construction inchoative ; au contraire, on peut la trouver un peu partout dans la phrase ou dans le texte :

Le jeune officier rougit et avoua, quelque peu confus : [...].

Cette question sembla causer à Mr Coleman un certain embarras. Il rougit légèrement [...]. ( A. Christie)

Rougissant légèrement, car je répugne à exposer ma manière de voir, je répliquai : [...]. (A. Christie)

Elle en rajouta en minaudant et en rougissant légèrement, comme si elle était embarrassée. (Harlequin)

Widziatem, że jest wściekly, zaczerwienit się na twarzy, dolna szczękę wysunąt do przodu... (J. Maślanek)

Zenek poczerwieniat, wyraz zawstydzenia czy zakłopotania przemkną po jego twarzy $i$ znikt. (I. Jurgielewiczowa)

Zażenowany, spięty, czerwieniąc się co chwila, usitowat pokryć zdenerwowanie dowcipkowaniem [...]. (M. Miller)

Le début de l'état décrit par une construction inchoative a une valeur momentanée mais il n'est pas obligatoire qu'il soit explicité dans la phrase. Le plus souvent il est inféré à partir d'un acte qui en est la cause ou à travers des adverbiaux compatibles. Cette indication du moment initial dans le texte fait effacer, dans les constructions à interprétation résultative le verbe devenir dont le rôle est de marquer de façon abstraite la borne gauche (initiation) de l'état en question. Dans les séquences réalisant le motif conceptuel « rouge-émotion » nous considérons donc l'adjectif rouge comme une forme abrégée de l'expression devenu rouge : 
Elle envoya une pique en italien à Orsetta pour lui faire savoir que son arrivée tombait au plus mauvais moment. Portant la main à ses lèvres, rouge de confusion, la gouvernante se confondit en excuses. (M. de Fontenay)

Po ósmej temperatura podskoczyła na nowo, kiedy na budowe wpadt z piskiem opon czerwony z pasji Markowski i zaczą dyskutowć z równie czerwonym kierownikiem, kto, komu i ile forsy przyniesie w zębach. (M. Czubaj, M. Krajewski)

3.3. L'explicitation textuelle de trois segments : réaction corporelle + l'émotion source de la réaction corporelle + cause de l'émotion (C)

Le troisième segment du motif conceptuel « rouge-émotion » porte sur la cause de l'émotion même. Encore une fois, cet élément du motif n'est pas impliqué sémantiquement, c'est une structure adjointe dont l'expression n'est pas obligatoire. On peut trouver des cas où tous les éléments du motif se rencontrent dans une phrase, mais le plus souvent ils se dispersent dans le texte. "Les phrases typographiques découpent des unités sémantiquement très inégales. On ne peut pas dire que chaque proposition est délimitée par le couple majuscule-point » (Adam, 1999, p. 53). On peut retrouver dans le texte l'explicitation de tous les segments du motif, comme dans les exemples suivants :

La pensée lui avait échappée avant qu'elle ait pu la refouler. Alison en fut choquée, ébranlée au plus profond de son être, et elle rougit violemment. (Harlequin)

To wspaniała dziewczyna, naprawdę, gratuluje pani córki. Kobieta zaczerwienita się z zadowolenia, a na nos i policzki wystapity jej piegi. (M. Nurowska)

La situation la plus fréquente consiste pourtant en une explicitation partielle du motif conceptuel «rougir-émotion». Le plus souvent, c'est l'explicitation de l'émotion qui manque. La question se pose de savoir comment, dans un texte, trouver le lien entre un événement et un état de façon à ce que cet événement soit à l'origine de l'état en question. La réponse située sur le niveau de description linguistique est simple : la construction inchoative doit repérer son point de référence - en même temps le début de l'état qu'elle dénote - dans le texte environnant. Comme il s'agit de la valeur momentanée de la cause, le moment initial de l'émotion sera identifié au moment de l'événement qui en est la source. C'est pour cette raison que le texte semble conserver dans ces cas la chronologie des faits : la cause est explicitée en premier lieu, la manifestation de l'émotion la suit.

L'identification de l'émotion n'est pas du même ordre. C'est un phénomène extralinguistique qui obéit aux facteurs subjectifs. On ne peut se fier ici qu'aux stéréotypes culturels dont certains, certes, ont trouvé leur expression dans la langue. Nous nous référons ici à la liste de E. Pilecka (2010), citée un peu plus haut : rougir de honte/confusion/colère/plaisir, etc. Les analyses de A. Kacprzak (2013) consa- 
crées aux formules rouge comme/czerwony jak semblent confirmer que les mêmes émotions dominent dans les comparaisons analysées. Pourtant, quand le lecteur est confronté à cette lacune dans un texte, c'est lui qui décide du type d'émotion non explicitée, n'empêche qu'il le fait sur la base du motif conceptuel qui, d'une part, permet d'inférer l'information qui manque et d'autre part assure la cohérence du texte lacunaire, comme dans les exemples suivants :

Elle pense que tu es le soleil de ma vie! Et je ne suis pas loin de penser la même chose! Kate rougit sous le compliment. (Harlequin)

Elle sembla soudain se rendre compte de sa tenue, car elle rajusta son peignoir, laissant son regard glisser sur le torse nu de Craig. Elle rougit, puis lui sourit. (N. Collins)

Podgladateś? - Lyl zaczerwienita się. - Gdzież bym śmiał. (I. Surmik)

My wcale nie jesteśmy, pani Anko, rodzinq. Ja zawsze pozostanę dla was intruzem.

Anna poczerwieniała, jakby złapano ją na gorącym uczynku. (T. Dołęga-Mostowicz)

\section{L'EXPRESSION DU MOTIF CONCEPTUEL « ROUGIR-ÉMOTION » DANS UN GENRE TEXTUEL}

Les analyses de textes et leur classification en genres discursifs ou en séries génériques proposent des conclusions valables à tous les textes réunis dans une série générique : ils seront tous formulés dans le même but, dans les mêmes conditions socio-historiques, par conséquent, pour être reconnus comme occurrences génériques, ils doivent avoir la même structure régulière et leurs propres dénotations. La forme régulière et le vocabulaire assorti garantissent la reconnaissance du genre et aboutit à une communication réussie. Partie de ce principe, nous postulons que, si un motif conceptuel est spécifique pour un genre, il doit y avoir une réalisation spécifique sous forme d'une structure lexico-syntaxique appropriée.

\subsection{Le roman sentimental}

Dans le roman sentimental, la réalisation la plus fréquente du motif conceptuel « rougir-émotion » met en jeux deux personnes de sexe opposé dont l'une rougit le plus souvent de confusion (aussi de honte et de désir). A vrai dire, elle reproduit une version stéréotypique de personnes timides et sincères qui ne sont pas suffisamment perfides pour cacher leurs vraies émotions. Elles réagissent surtout aux paroles qui leur sont adressées ou aux comportements (ne serais-ce qu'une simple apparition) de la personne impliquée. Il faut mentionner toutefois que, globalement, dans le corpus de romans sentimentaux, ce motif n'est pas très représenté. Dans leur analyse de clichés dans les romans sentimentaux D. Legallois, T. Charmion et T. Poibeau 
(2016) ne le citent pas parmi les constructions les plus fréquentes. Toutefois, il y est présent, et, ce qui est intéressant, atteste un grand nombre de constructions à valeur résultative ou télique qui se focalisent sur le nouvel état :

Il sentit une bouffée de tendresse. Elle se sentit rougir sous ce regard brûlant et inquisiteur qui semblait chercher à la mettre à nu, l'envahir avec, dans les yeux, une étrange lueur. (Harlequin)

Une flambée de désir le terrassa. - Ce compliment me va droit au cour, balbutia-telle, en rougissant. (Harlequin)

April [...] przez moment gapiła sie otwarcie na jego szczuple biodra i tors, który zdawat się prężyć w wyrazie asertywności, tupetu albo jeszcze czegoś innego, czego nie potrafita zdefiniować. Właśnie zaczęla się czerwienić, gdy jej spojrzenie padło na zwisającego $z$ ust papierosa. (M. Gable)

Sam poczerwieniat. Jej śmiech wywołat w nim falę goraca. (Harlequin)

\subsection{Le roman policier}

Contrairement à toute attente, rougir ne semble pas jouer un rôle important dans ce genre textuel et surtout dans sa partie cruciale qu'est l'interrogatoire. Dans les romans policiers modernes, il est presque inexistant ou marginal. On peut pourtant trouver des fragments où la confusion ou la colère de la personne interpelée apporte une information pertinente pour un roman policier. La version appropriée du motif conceptuel dans ce cas pourrait être décrite comme une réaction spontanée de confusion à une question de fond de la part de l'enquêteur et vérifiant la vérité des constats précédents, comme dans les exemples ci-dessous :

Mais Mr Carey n'a-t-il pas quitté le chantier? Et Mr Coleman se trouvait-il réellement à Hassanieh à l'heure où le meurtre fut commis? Bill Coleman rougit, ouvrit la bouche, la referma et jeta un regard embarrassé autour de lui. Mr Carey ne changea point d'expression. (A. Christie)

Skąd wiesz? - napadt na niego na nowo rozsierdzony Cal. A może to ty pomogłeś jej w ucieczce? Jarik zaczerwienit się po uszy. Czy ona była więźniem, panie? (I. Surmik)

Pater spojrzat na czterdziestolatka z wypiekami na twarzy. Nazywam się Jarosław Pater. Komenda Wojewódzka. Pan jest właścicielem? Mężczyzna poczerwieniał jeszcze bardziej i skinąt głowa. Nazywam się Krzysztof Markowski, a to mój wspólnik, Rafat Misiak. Nadkomisarz przypatrywat się mężczyznom. Misiak nieco cofnąt się za wspólnika. (M. Czubaj, M. Krajewski)

Toutefois, le plus souvent, le motif de «rougir-émotion» s'actualise dans le roman policier dans la description de personnages. Il fait partie de l'image totale que l'auteur offre au lecteur de l'un de ces acteurs. Il construit son personnage selon un 
modèle psychologique de réactions prototypiques en laissant au lecteur le soin de reconnaitre le type proposé avec tout le bagage de réactions qu'il est censé avoir. Le plus souvent, ce motif reproduit celui du stéréotype culturel de l'époque en éliminant des réaction du type désir, plaisir ou pareilles. Parfois elles apparaissent, mais là, on est loin du motif conceptuel propre au roman policier. Il est question plutôt d'un emprunt générique (Muryn, Niziołek, 2017) qui permet au lecteur du roman policier d'activer ses compétences, peut-être, de lecteur de romans psychologiques, de romans sentimentaux, de discours journalistiques, etc., dont il tire ses compétences interprétatives. La question reste ouverte, une analyse détaillée de ces discours y apportera peut-être une réponse décisive. Pour l'instant, toutefois, nous devons nous fier aux faits textuellement attestés et ils confirment que le lecteur, confronté à une réaction du type rougir, si sa valeur émotionnelle n'est pas explicitée, est guidé par ses convictions pré-acquises et qu'il suit une sorte de code : bon/mauvais : réaction (surtout confusion, ensuite colère). A notre sincère regret, il est impossible, pour l'instant, de repérer les motifs conceptuels de victime ni de coupable se reconnaissant à l'aide du langage corporel «rougir-émotion» dans le roman policier. Cette simple réaction n'est pas suffisamment exploitée dans ce genre textuel. Il suffit de dire que dans le corpus de 47 romans policiers le verbe rougir n'apparaît que dans 38 occurrences.

\section{UNE CONCLUSION?}

A cette étape de la recherche, il est trop tôt pour conclure sur la question de savoir comment le savoir sur le langage non verbal peut influencer les genres textuels et comment ils en profitent. Nous avons réduit notre recherche à une seule manifestation de l'émotion, celle de rougir. La première conclusion qui se dessine sur la base de cette analyse est que, dans les romans sentimentaux et selon la réalisation du motif conceptuel "rougir-émotion», les hommes et les femmes rougissent toujours de confusion ou de désir à la vue ou aux propos de leurs partenaires, mais cette réaction n'est plus évidente quand ces personnages sont inculpés dans une affaire criminelle. On dirait, si l'on accepte la littérature comme miroir de la réalité, que les hommes aient su maîtriser ce qu'on appelle un langage involontaire du corps, ou bien que leur code moral soit plus ouvert que pour les générations précédentes. Les réalisations du motif « rougir-émotion » pertinent pour le roman policier sont plutôt marginales est marquées par le temps: le roman moderne ne semble pas attacher une importance à cette réaction corporelle et, par conséquent, faire preuve de la réalité : certaines émotions ne font plus rougir.

Toutefois, il semble incontestable que la valeur aspectuelle des expressions explicitant les segments du motif « rougir-émotion » joue un rôle décisif pour deux raisons : premièrement, par le jeu des inférences, elle permet d'assurer la cohérence 
du segment textuel qui dépasse le cadre de la phrase (p. ex. le verbe inchoatif présuppose la cause sans être obligé de l'expliciter); et deuxièmement, la valeur aspectuelle d'une construction oriente la lecture du segment textuel vers le plan des événements et des actions (le premier plan de H.Weinrich, 1973) ou vers l'arrière plan de la narration : les formes qui se focalisent sur l'effet sont propres à l'arrièreplan, tandis que les formes qui se focalisent sur le moment initial sont propres au premier plan. Les unes participent à la description (p. ex. la construction du personnage), les autres se concentrent sur la succession de faits. Chaque genre les exploite à sa façon, mais il nous semble que certaines observations sont justifiées : le roman sentimental qui opte pour la valeur résultative, préfère décrire le personnage ; le roman policier moderne (si jamais il en profite) situe la réaction de rougir aussi à l'arrière-plan. C'est chez des auteurs classiques comme A. Christie ou G. Simenon qu'on peut trouver encore les segments textuels ou le motif «rougir-émotion» se trouve au premier plan :

Vous vous passionnez pour l'archéologie? Cette question sembla causer à Mr Coleman un certain embarras. Il rougit légèrement et lança au Dr Leidner le regard confus d'un écolier pris en faute. (A. Christie)

- Il le faisait pour s'en débarrasser, pour se mettre en règle avec sa conscience... - Sa conscience ?... Martin rougit, fut plus embarrassé. - Il a eu des torts envers Juliette, n'est-ce pas ? dit-il à voix plus basse. - Juliette ? [...] Qu'est-ce qu'il a fait pour elle?... Rien !... [...] - Il ne lui a rien donné, évidemment !... [...] Le visage de Martin s'était empourpré. Maigret le regardait avec étonnement, avec pitié. (G. Simenon) 


\section{BIBLIOGRAPHIE}

Adam, J.-M. (1999). Linguistique textuelle. Des genres de discours aux textes. Paris : Nathan.

Adam, J.-M. (2008). Linguistique textuelle. Introduction à l'analyse textuelle des discours. Paris : Armand Collin.

Ciszewska, E. (2002). Expression de la perfectivité en français contemporain. Katowice : Wydawnictwo Uniwersytetu Śląskiego.

Dufays, J.-L. (1994). Stéréotype et lecture, éd. Mardaga, coll. « Philosophie et langage ».

Ekman, P. (2018). Kłamstwo i jego wykrywanie $w$ biznesie, polityce i malżeństwie. Warszawa : PWN.

Grossmann, F. (2017). « Du rituel social aux motifs : la scène de présentation interpersonnelle dans le roman ». Synergies Pologne, 14, 13-29.

Grzmil-tylutki, H. (2016). "Initiation à la linguistique textuelle ». In : J. Górnikiewicz, B. Marczuk, I. Piechnik (ed.), Études sur le texte dédiées à Halina Grzmil-Tylutki. Kraków : Biblioteka Jagiellońska, 15-60.

Guiraud, P. (1980). Le langage du corps, Que sais-je ? Paris : PUF.

Kacprzak, A. (2013). « A la recherche de l'équivalence interlinguale : rouge comme... vs czerwony jak burak... ». Roczniki Humanistyczne, 8, 25-40.

Karolak, S. (2001). « Czy aspekt jest kategorią gramatyczną? ». In : S. Karolak, Od gramatyki do semantyki. Warszawa : Instytut Slawistyki PAN, 461-475.

Karolak, S. (2001a). « Inchoatywność i rezultatywność w języku bułgarskim ». In : S. Karolak, Od gramatyki do semantyki. Warszawa : Instytut Slawistyki PAN, 637-653.

Krejdlin, G. (2008). « Le langage du corps et la gestuelle (kinésique) comme champs de la sémiotique non-verbale : idées et résultats ». Cahiers Slaves, 9, 1-23.

Krzyżanowska, A. (2006). " Objawy smutku utrwalone w języku (polsko-francuska analiza porównawcza) ». In : K. Michalewski (ed.), Wyrażanie emocji. Łódź : Wydawnictwo UŁ, 561-572.

Legallois, D., Charnois, T., Poibeau, T. (2016). « Repérer les clichés dans les romans sentimentaux grâce à la méthode des motifs ». Lidil, revue de linguistique et de didactique des langues, 53, 95-117.

Legallois, D., Tutin, A. (2013). «Présentation : vers une extension du domaine de la phraséologie ». Langages, 189, 3-25.

Longrée, D., Mellet, S. (2013). «Le motif : une unité phraséologique englobante ? Etendre le champs de la phraséologie de la langue au discours ». Langages, 189, 65-79.

Muryn, T., Niziołek, M. (2016). " Pour une analyse phraséologique du roman policier ». In : Mogorron Huerta, P., Cuadrado Rey, A., Martinez Blasco, I., Navarro Brotons, L. (ed.), Fraseologia, variacion y traducción. Frankfurt am Main : Peter Lang.

Muryn, T., Niziołek, M. (2017). «L'intertexte dans le roman policier ». Synergies Pologne, 14, 61-72.

Muryn, T., Niziołek, M., Hajok, A., Prażuch, W., Gabrysiak, K. (2016). « Scène de crime dans le roman policier : essai d'analyse lexico-syntaxique ». In : Actes du CMLF2016, http://dx. doi.org/10.1051/shsconf/20162706007.

Novakova, I., Sorba, J. (2017). « A la recherche des motifs autour de meurtre, crime, assassinat dans le roman policier contemporain ». Synergies Pologne, 14, 29-43.

Pilecka, E. (2010). Verbes intensifieurs et leur fonctionement en français contemporain. Lask : LEKSEM.

Rastier, F. (2001). Eléments de théorie des genres, Texte diffusé sur la liste fermée « Sémantique des textes ».

Śmiech, W. (1986). Derywacja prefiksalna czasowników polskich. Łódź : Ossolineum. 
Tutin, A., Grossmann, F. (2014). L'écrit scientifique : du lexique au discours. Autour de Scientext. Rennes : Presses de l'Université de Rennes.

Weinrich, H. (1973). Le Temps, le récit et le commentaire, trad. fr. M. Lacoste. Paris : Seuil.

Wierzbicka, A. (1999). Emotions accross Languages and Cultures. Diversity and Universals. Paris / Cambridge : Cambridge University Press / Editions de la Maison des Sciences de l'Homme.

Włodarczyk, A., Włodarczyk, H. (2001). « La préfixation verbale en polonais II. L’aspect perfectif comme hypercatégorie ». Etudes cognitives, 4, 111-121. 